\title{
Optimal landing position in reading isolated words and continuous text
}

\author{
F. VITU, J. K. O'REGAN, and M. MITTAU \\ Groupe Regard, Laboratoire de Psychologie Expérimentale, CNRS \\ Université René Descartes, EHESS, EPHE, Paris, France
}

\begin{abstract}
During isolated-word reading, within-word eye-movement tactics (i.e., whether the eye makes one or more fixations on the word) depend strongly on the eye's first fixation position in the word; there exists an optimal landing position where the probability of having to refixate the word is much smaller than when the eye first fixates other parts of the word. The present experiment was designed to test whether the optimal landing position effect still exists during text reading, and to compare the nature and strength of the effect with the effect found for isolated words. The results confirmed the existence of an optimal landing position in both reading conditions, but the effect for words in texts was weaker than it was for isolated words, probably because of the presence of factors such as reading rhythm and linguistic context. However, the effect still existed in text reading; within-word tactics during text reading are dependent on the eye's initial landing position in words. Moreover, individual fixation durations were dependent on withinword tactics. Thus, the initial landing position in words must be taken into account if one wishes to understand eye-movement behavior during text reading. Further results concerned the effects of word length and word frequency in both reading conditions.
\end{abstract}

A large number of experiments in recent years have been done to investigate the factors that determine fixation positions and fixation durations in reading. As concerns fixation positions, the preferred landing position in words is generally located at the middle or at the beginning of words (Dunn-Rankin, 1978; McConkie, Kerr, Reddix, \& Zola, 1988; Rayner, 1979), but it depends on several factors. McConkie et al. (1988), in a very detailed study, and O'Regan (1979) have shown how landing positions in words depend on the length of the word the eye is leaving, the length of the word the eye is saccading towards, and the position relative to a word from which the eye's movement is launched. Although many experimenters have studied the influence of linguistic factors on the probability of fixating a word during reading (e.g., Balota, Pollatsek, \& Rayner, 1985; Ehrlich \& Rayner, 1981; Zola, 1984), there is only weak evidence that linguistic factors determine where the eye lands next (for a review of this question, see O'Regan, 1990).

Fixation durations have been shown to depend on the length (Just \& Carpenter, 1987) and frequency (Inhoff \& Rayner, 1986; Rayner \& Duffy, 1986) of the fixated word, on the amount of available parafoveal information (Balota et al., 1985; Lima \& Inhoff, 1985; McConkie, Underwood, Zola, \& Wolverton, 1985; Rayner, 1975; Rayner, Inhoff, Morrison, Slowiaczek, \& Bertera, 1981),

We wish to thank $K$. Rayner, T. Nazir, and two anonymous reviewers for helpful comments. Correspondence may be addressed to either F. Vitu or J. K. O'Regan, Groupe Regard, Laboratoire de Psychologie Expérimentale, CNRS, Université René Descartes, 28 rue Serpente, 75006 Paris, France. and on the word's predictability given its preceding context (Ehrlich \& Rayner, 1981; Zola, 1984). Hogaboam (1983) suggested that fixation durations might also be influenced by the tactics of the eye as it moves within the word; when a fixation is the only one in a word, its duration may be different than when it is followed by other fixations within the word.

In a recent strategy-tactics theory, O'Regan (1990) has suggested that the variability of landing positions and fixation durations in reading might be explainable partly in terms of a newly discovered phenomenon in the recognition of isolated words: the phenomenon that eyemovement behavior during the reading of isolated words depends strongly on the position in the word that the eye first fixates (O'Regan, 1984, 1989, 1990; O'Regan \& Lévy-Schoen, 1987; O'Regan, Lévy-Schoen, Pynte, \& Brugaillere, 1984). There exists a position, called the optimal landing position, at which the probability that the eye will refixate the word is smaller than it is when the eye first fixates other places in the word instead. The total time spent fixating an isolated word (gaze duration) is also shortest when the eye starts at the optimal position. Furthermore, depending on whether one, two, or more fixations are made within the word, different patterns of fixation durations will be observed. The location of the optimal landing position depends on lexical aspects of the word (Holmes \& O'Regan, 1987), but it is generally near the word's middle. Note that in earlier work (O'Regan \& Lévy-Schoen, 1987; O'Regan et al., 1984), the term "convenient viewing position" was used instead of optimal landing position. We now prefer the latter term, because it emphasizes the idea that there is a "best place" 
to fixate within words (from the point of view of minimizing the number of fixations or the gaze duration), although the eye may actually "prefer"' to land elsewhere.

A further step toward an understanding of the variability of eye-movement parameters in reading would be to show that the optimal landing position phenomenon exists not only for the reading of isolated words, but also for the reading of continuous text. However, various factors in the reading of continuous text might modify oculomotor behavior, and thereby might modify the optimal landing position effect. First, in the text-reading situation, the eye is engaged in a dynamic sequence of forward movements with their accompanying attentional shifts (in contrast with the isolated-word-reading situation, which is much more static, and in which subjects focus all their attention in the first part of each trial on the fixation point). This reading rhythm involved in the text-reading situation could globally decrease the probability of refixating words and thus weaken the optimal landing position phenomenon. Second, linguistic context, by facilitating word recognition (cf., e.g., Balota \& Rayner, 1983), might decrease the need to refixate words (Balota et al., 1985), and thus it too might weaken the optimal landing position effect. Finally, parafoveal preprocessing might also have a similar weakening effect (Balota et al., 1985; Balota \& Rayner, 1983; Inhoff \& Rayner, 1980; McClelland \& O'Regan, 1981; Rayner, McConkie, \& Ehrlich, 1978; Rayner, Well, Pollatsek, \& Bertera, 1982). In fact, because parafoveal preprocessing allows the first few letters of a word to be previewed, it might also cause the optimal position to be displaced to the right. This is suggested by results obtained by Vitu and O'Regan (1988, in press) in an experiment in which the possibility of parafoveally preprocessing isolated words was manipulated. Although parafoveal preprocessing did not appreciably weaken the optimal landing position phenomenon (though it globally reduced refixation probability and gaze duration), it shifted the optimal landing position to the right for long words.

Recently, McConkie, Kerr, Reddix, Zola, and Jacobs (1989; see also Blanchard \& McConkie, cited in O'Regan \& Lévy-Schoen, 1987) did observe an optimal landing position phenomenon in the reading of normal text. Instead of forcing the eye to fixate words in particular positions, as was done for isolated words by $O$ 'Regan et al. (1984), McConkie et al. (1989) measured the probability of refixating a word as a function of the positions where the eye spontaneously landed during normal reading. As O'Regan et al. (1984), did for isolated words, they found that there was an optimal landing position at which the probability of refixating the word was smaller than it was when the eye first fixated another position in the word. However, no analysis was presented concerning the influence of first fixation position on gaze durations or individual fixation durations. Furthermore, no attempt was made to compare the optimal landing position phenomenon in the two situations of isolated-word as opposed to text reading. However, if it were the case that the optimal landing position effect is indeed weaker in normal reading as compared with isolated word reading, or if the optimal landing position is in a different location, this would suggest the action on the optimal landing position phenomenon of factors present during normal reading, such as reading rhythm, parafoveal preprocessing, or linguistic context. In other words, a comparison between normal and isolated-word reading would tell us how initial landing position in words interacts with reading rhythm, parafoveal preprocessing, and linguistic context to determine eye-movement behavior during reading.

In the present experiment, we made a direct comparison of the optimal landing position phenomenon in two situations: the reading of isolated words and the reading of continuous texts. The same corpus of words was used in both cases: Half the subjects saw half the corpus in the text-reading situation and the other half in the isolated word situation; the other half of the subjects saw the reverse. We measured refixation probability, gaze durations, and individual fixation durations, as a function of landing position.

\section{METHOD}

\section{Subjects}

Twenty subjects participated. They were between 20 and 30 years old. All were unfamiliar with the equipment and the purpose of the experiment.

\section{Materials}

For the text-reading condition, 1620 -line texts were selected from a book of amusing stories about the history of Paris. For each word length $(5,6,7,8$, and 9 letters), 160 test words were defined, such that there were about 10 words of each length in each text. The test words were never the first or the last in each 80-character line, and they were located in the central zone of 60 characters, where the eye-movement recording accuracy was best. In order to control for repetition effects, if 1 of the selected test words occurred more than once in a given text, it was selected at its first occurrence in this text. Moreover, a given word was a test word in only one of the 16 texts. For each word length, test words were classified into three frequency groups: low, medium, and high, as defined by the Trésor de la langue française count of 38 million words; it was only approximately possible to balance the means of these frequency ranges across all word lengths, because high-frequency long words and low-frequency short words that fit into the texts were hard to find. Table 1 shows the exact means used.

The texts were divided into two groups of eight texts, each group containing 400 test words ( 80 test words for each of the five lengths). Two 400-word lists, containing the same test words as used in the two groups of texts, were constructed for the isolated-word-reading

Table 1

Means of Frequency Ranges (in Occurrences per Million) for Each Word-Frequency Category and Each Word Length

\begin{tabular}{cccr}
\hline & \multicolumn{3}{c}{ Frequency } \\
\cline { 2 - 4 } Word Length & Low & Medium & High \\
\hline 5 letters & 8 & 61 & 986 \\
6 letters & 7 & 67 & 1031 \\
7 letters & 6 & 48 & 391 \\
8 letters & 5 & 44 & 472 \\
9 letters & 6 & 50 & 331 \\
\hline
\end{tabular}


condition. For each test word, an additional comparison word or group of words was defined such that the sequence of test word and comparison word(s) formed a simple phrase that was always syntactically correct but was only in half of the cases semantically correct (e.g., chats followed by de gouttiere (alley cats) versus chiens followed by en skis (dogs on skis).

\section{Design}

Two reading conditions were used: reading of isolated words and reading of continuous texts. For each condition, there were two groups of test words, containing 80 words of each length $(5,6$, 7,8 , and 9 letters). Table 2 shows the number of words in each of the frequency categories for each length; this could only be counterbalanced approximately, because of the difficulty of creating texts with rare short words and frequent long words. Half of the subjects saw one test-word group in the isolated-word-reading condition and the other group in the text-reading condition. For the other half of the subjects, the attribution of test-word groups to reading conditions was reversed. Each subject saw each test word only once. The reading condition order was counterbalanced between subjects: Half of the subjects read the continuous texts first and then read the isolated words, and the other half did the conditions in the opposite order. The order in which the subjects read the texts was randomized for each subject. The order of the test words in the isolated word condition was also randomized for each subject.

In the isolated-word-reading condition, the imposed first fixation position in words was manipulated. A word could be presented so that the eye first fixated the middle of one of five equal zones in the word (for example, for 5-letter words, the zones were 1 letter wide; for 9-letter words, they were 1.8 letters wide). Both groups of 80 words in each word-length category were divided into five subgroups of 16 words, and the two subject groups were also divided into five subgroups of 2 persons. A Latin square was used to determine which words were attributed to which first fixation zone for which subject. For each word length, each subject saw 16 words presented first at each of the five zones. Over all the subjects, each word was seen an equal number of times from each first fixation zone. Unfortunately, the number of words in a given frequency $x$ length $x$ first fixation zone cell could not be perfectly balanced (see Table 3).

In the text-reading condition, first fixation position in words could of course not be manipulated a priori, so this was not strictly an independent variable. Nevertheless, in the analysis of the textreading data, fixations were classified according to which of the five zones in a word the eye first fell on, and analyses of variance were done as for isolated words. The number of measurements available for the five zones was not balanced in this case, however.

\section{Procedure}

The subjects sat in an adjustable chair, their eyes $50 \mathrm{~cm}$ from the computer screen. The text-reading condition required accurate eye-movement recording over the 80 -character screen width $\left(22^{\circ}\right)$, so a bite bar was used in this condition in order to minimize head movements. For the isolated-word-reading condition, the eye-

Table 2

Number of Words Presented in Both Isolated-Word-Reading and Text-Reading Conditions for Each Word Frequency and Word Length

\begin{tabular}{cccc}
\hline & \multicolumn{3}{c}{ Frequency } \\
\cline { 2 - 4 } Word Length & Low & Medium & High \\
\hline 5 letters & 24 & 73 & 63 \\
6 letters & 28 & 86 & 46 \\
7 letters & 42 & 83 & 35 \\
8 letters & 61 & 72 & 27 \\
9 letters & 68 & 78 & 14 \\
\hline
\end{tabular}

Table 3

Number of Words in Each of the Five Possible First Fixation Positions in the Word in the Isolated-Word-Reading Condition, for Each Word Frequency and Word Length

\begin{tabular}{|c|c|c|c|}
\hline \multirow[b]{2}{*}{ Position } & \multicolumn{3}{|c|}{ Frequency } \\
\hline & Low & Medium & High \\
\hline \multicolumn{4}{|c|}{ 5-Letter Words } \\
\hline Zone 1 & 6 & 14 & 12 \\
\hline Zone 2 & 4 & 16 & 12 \\
\hline Zone 3 & 6 & 13 & 13 \\
\hline Zone 4 & 4 & 15 & 13 \\
\hline Zone 5 & 4 & 15 & 13 \\
\hline \multicolumn{4}{|c|}{ 6-Letter Words } \\
\hline Zone 1 & 5 & 19 & 8 \\
\hline Zone 2 & 6 & 18 & 8 \\
\hline Zone 3 & 6 & 16 & 10 \\
\hline Zone 4 & 6 & 16 & 10 \\
\hline Zone 5 & 5 & 17 & 10 \\
\hline \multicolumn{4}{|c|}{ 7-Letter Words } \\
\hline Zone 1 & 8 & 17 & 7 \\
\hline Zone 2 & 9 & 17 & 6 \\
\hline Zone 3 & 8 & 16 & 8 \\
\hline Zone 4 & 8 & 17 & 7 \\
\hline Zone 5 & 9 & 16 & 7 \\
\hline \multicolumn{4}{|c|}{ 8-Letter Words } \\
\hline Zone 1 & 12 & 15 & 5 \\
\hline Zone 2 & 12 & 14 & 6 \\
\hline Zone 3 & 12 & 14 & 6 \\
\hline Zone 4 & 12 & 16 & 4 \\
\hline Zone 5 & 13 & 13 & 6 \\
\hline \multicolumn{4}{|c|}{ 9-Letter Words } \\
\hline Zone 1 & 14 & 15 & 3 \\
\hline Zone 2 & 14 & 16 & 2 \\
\hline Zone 3 & 13 & 16 & 3 \\
\hline Zone 4 & 14 & 15 & 3 \\
\hline Zone 5 & 13 & 16 & 3 \\
\hline
\end{tabular}

movement recording zone was the same length as the test words (a maximum of 9 characters), and each trial involved recording for only a very short time ( $\leq .5 \mathrm{sec}$ ), so it was only necessary to stabilize the subjects' heads with a chin/forehead rest.

The isolated-word-reading condition began with a calibration phase, during which the recording system was adjusted for a perfect correspondence between the recorded fixation position and the fixated target (in a central zone of 30 characters). When calibration was correct, the first trial began. Two vertically aligned line segments with a letter-high gap between them appeared at the center of the screen, and as soon as the subject fixated in the gap ( \pm .125 characters from the gap), the fixation lines were replaced by a group of words (the test word and comparison words). The test word appeared at different positions relative to the gap in the fixation lines, such that the directly fixated position in the word was at the middle of one of the five possible first fixation zones. Whatever the position of the test word relative to the fixation point, the comparison word(s) was always placed beginning 1 character space after the test word. During fixation of the test word, it alone was visible, the comparison word(s) being masked. When the computer detected that the eye had passed the middle of the space separating the test word and the comparison word(s), the test word was masked (it then remained masked until the end of the trial), and the comparison word(s) became visible. The subjects' task was to compare the test word to the comparison word(s) and to determine, by pressing a button, whether the whole sequence formed a semantically correct phrase. Reading of the 400 test words was done in four parts, 
interrupted by one or two pauses, and was preceded by 12 training trials.

In the text-reading condition, reading was also preceded by a calibration phase. Because our recording system was nonlinear for large eccentricities, this phase was divided into two parts. First, the recording system was adjusted in order to obtain an approximate correspondence between the recorded and actual fixation positions (in a central zone of 50 characters); then, a small target that the subject had to fixate appeared successively at six equally spaced positions covering the whole width of the screen. Linear interpolation between adjacent measurements was used to obtain accurate eye-position estimates during subsequent recording. After the calibration phase, two small vertically aligned fixation lines with a letter-high gap between them appeared on the left of the screen; when the subject fixated precisely in the gap $( \pm .25$ characters from the gap), the first line of the text appeared, with the third letter of the line at the gap position. When the subject had finished reading the line, he or she pressed a button and a new fixation mark appeared on the right of the screen. If the subject fixated it with an accuracy of better than \pm 1.5 letters, a fixation mark again appeared on the left of the screen and the sequence of events was repeated. With this procedure, the interval between finishing one line and starting to read the next line was not more than about $1 \mathrm{sec}$; usually, it was less. However, whenever a fixation on the righthand mark was not accurate, a whole new calibration phase was interposed. Since this caused an interruption of several seconds, making reading somewhat unnatural, we decided to reject all the data for a whole text if recalibration occurred more than three times during reading of the text. At the end of each text-reading session, the subjects were asked several questions about the text. The reading of the eight texts was preceded by the reading of a training text.

\section{Apparatus}

Eye movements were monitored by a photoelectric, infrared, iris/sclera boundary-detection technique. Eye position was sampled by a BBC computer every $10 \mathrm{msec}$. Saccade size and fixation duration were analyzed by the computer, according to the following principle: A saccade was defined as a change in eye position of more than .5 characters, taking less than $70 \mathrm{msec}$ and giving rise to a fixation lasting more than $70 \mathrm{msec}$. In both conditions (isolatedword and text reading), stimuli were presented on a green and white Zenith video monitor, refreshed every $20 \mathrm{msec}$. Characters were defined in an $8 \times 8$ pixel matrix that subtended $.3^{\circ}$. For isolated words, only lowercase characters were used, and for words in texts, upper- and lowercase characters were used as in normal print. A maximum of 80 characters could occur on any line.

\section{RESULTS}

The selection of data for the analysis was done according to the following criteria: in both the isolated-wordand the text-reading conditions, gaze durations on a test word were defined as being the sum of all individual fixation durations occurring on the test word; refixations of the test word that occurred after the eye had left the test word were not included in this sum. In the text-reading condition, first fixations on a test word were only considered when they occurred after a progressive saccade originating from a region to the left of the word. In both isolated-word- and text-reading conditions, we excluded cases in which individual fixation durations were less than $70 \mathrm{msec}$ or gaze durations lay outside two standard devi- ations from the mean of the given subject's gaze-duration distribution. Particular problems occurred in the textreading condition when subjects were unable to maintain accurate calibration while reading a text. As mentioned above, in this case, all the data for that subject reading that text were rejected. This occurred on one out of the eight texts for 4 subjects, and on two texts for 2 subjects.

The accuracy of eye-position recording was good in the isolated-word-reading condition, since measurements were made in the region where calibration was verified before each trial (that is, at the imposed fixation location in the word). In this case, since imposed calibration accuracy at the fixation point was \pm .125 letters, and measurements were taken up to only a few hundred milliseconds after that moment, accuracy was certainly much better than 1 letter.

In the text-reading condition, the imposed calibration accuracy was \pm .25 letters on the left of each line, and \pm 1.5 letters on the right. Because test words only appeared in the middle section of each line, an average level of accuracy of \pm 1 letter seems a reasonable estimation. Note that assurance that accuracy was sufficient for our purposes comes from the distribution of preferred landing positions in the test words (see Figure 1a-1e), which is similar to the distributions observed by McConkie et al. (1988). A further guarantee comes from the fact that, as we shall see, significant effects of the eye's position in a word were found; no such effects could have been found had accuracy been insufficient to distinguish the different positions.

\section{Isolated Words}

Probability of refixating a word. The probability of refixating a word in the isolated-word-reading condition depended strongly on the eye's first fixation position in the word (Figure $2 a$, top curves). There was an optimal landing position near the middle of each word; when the eye first fixated a word's middle, the probability of refixating within the word was much smaller than when the eye first fixated other positions in the word. This was confirmed by the analyses of variance, which showed a significant effect of initial fixation position for each word length $[F(4,64) \geq 9.31, p<.0005$, for each of the word lengths $5,6,7,8$, and 9 letters].

The probability of refixating a word was also affected by word length $[F(4,64)=56.551, p<.0005]$, as can be seen from the vertical displacement of the different curves in Figure 2a (top curves), where the data from all frequencies have been collapsed together, and as can be seen from Figure 3a (top curves), where the data have been collapsed over all initial fixation positions and plotted separately for high, medium, and low frequencies.

As is shown by the top curves in Figure 3a, which run closely together, the effect of word frequency on refixation probability was not globally significant $[F \mathrm{~s}(2,32)<$ 1, n.s., for 5-, 6-, 7-, and 8-letter words]. However, for 
DISTRIBUTION OF LANDING POSITIONS IN WORDS 5 - letter words

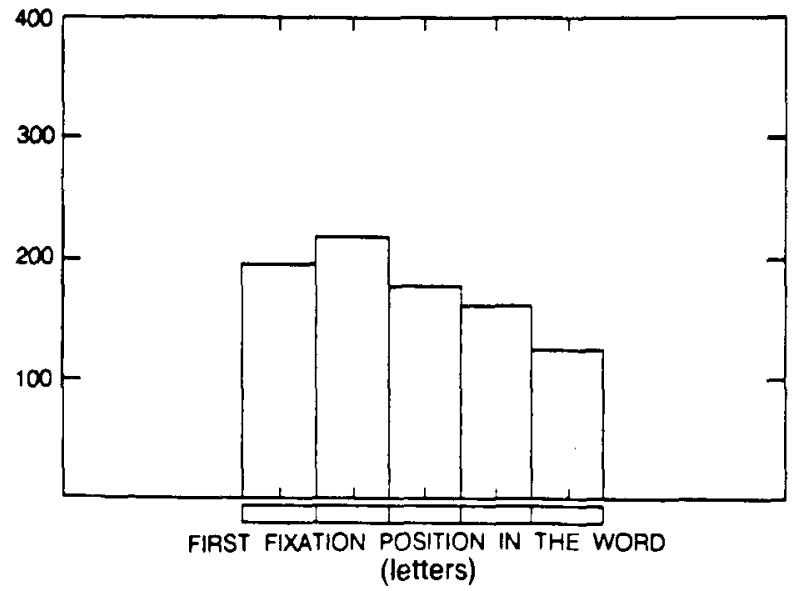

OISTRIBUTION OF LANOING POSITIONS IN WOROS 7 - letter words

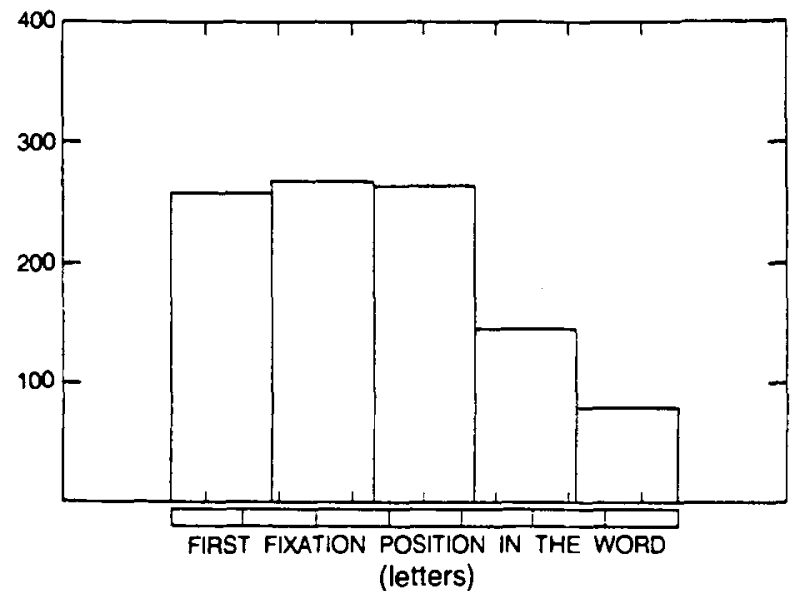

DISTRIBUTION OF LANDING POSITIONS IN WORDS 6- letter words

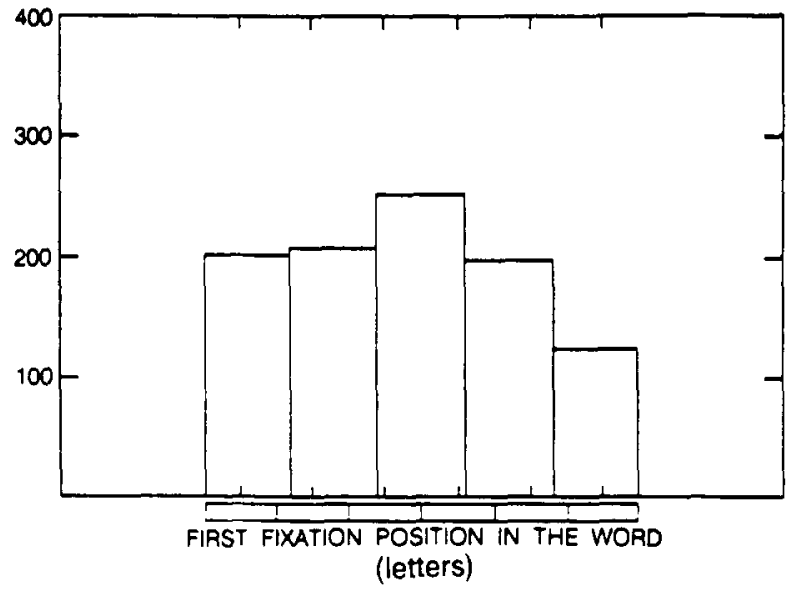

DISTRIBUTION OF LANCING POSITIONS IN WORDS 8 - letter words

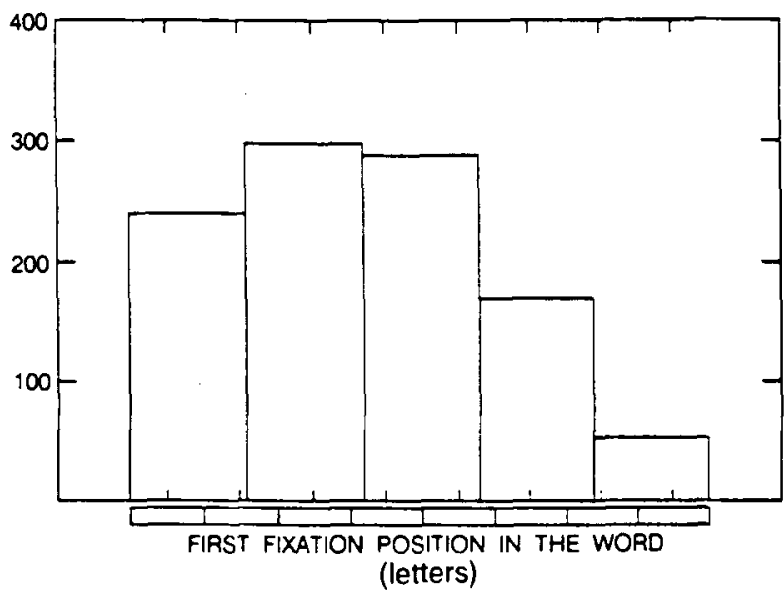

DISTRIBUTION OF LANDING POSITIONS IN WORDS 9 - letter words

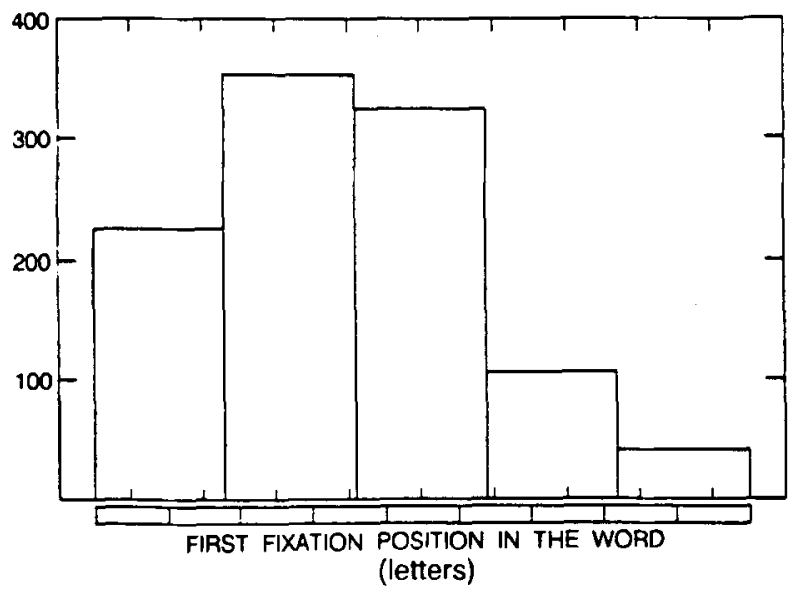

Figure 1. Number of fixations initially landing in each of the five zones in the test word, for 5-, 6-, 7-, 8-, and 9-letter words, in the text-reading condition. 


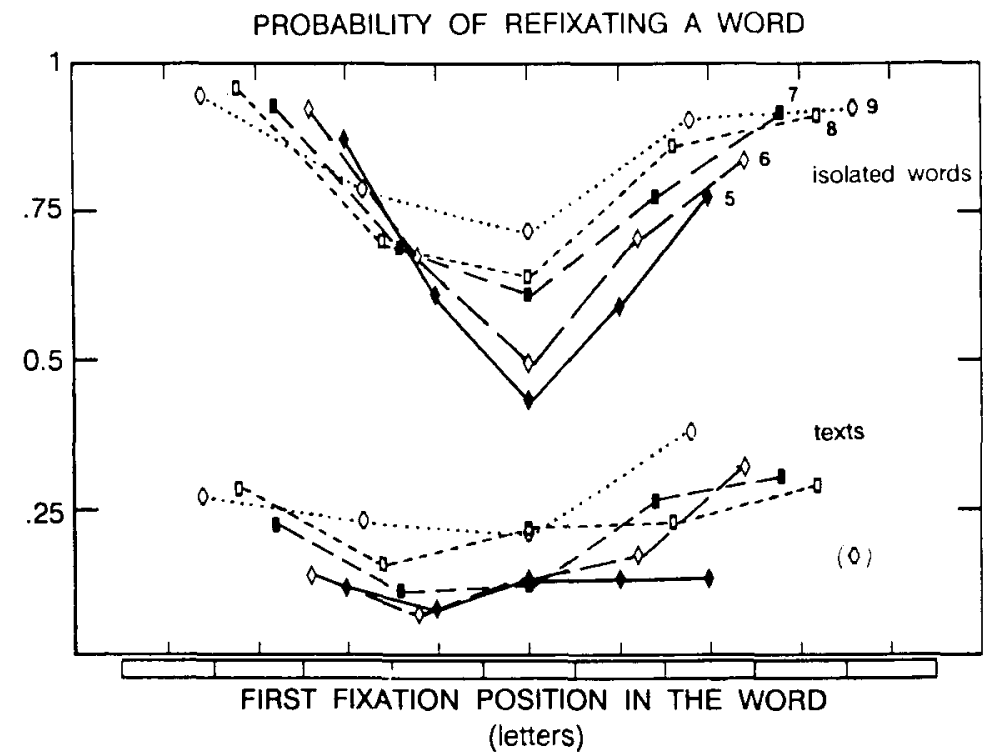

(a)

GAZE DURATION ON THE WORD (MSEC)

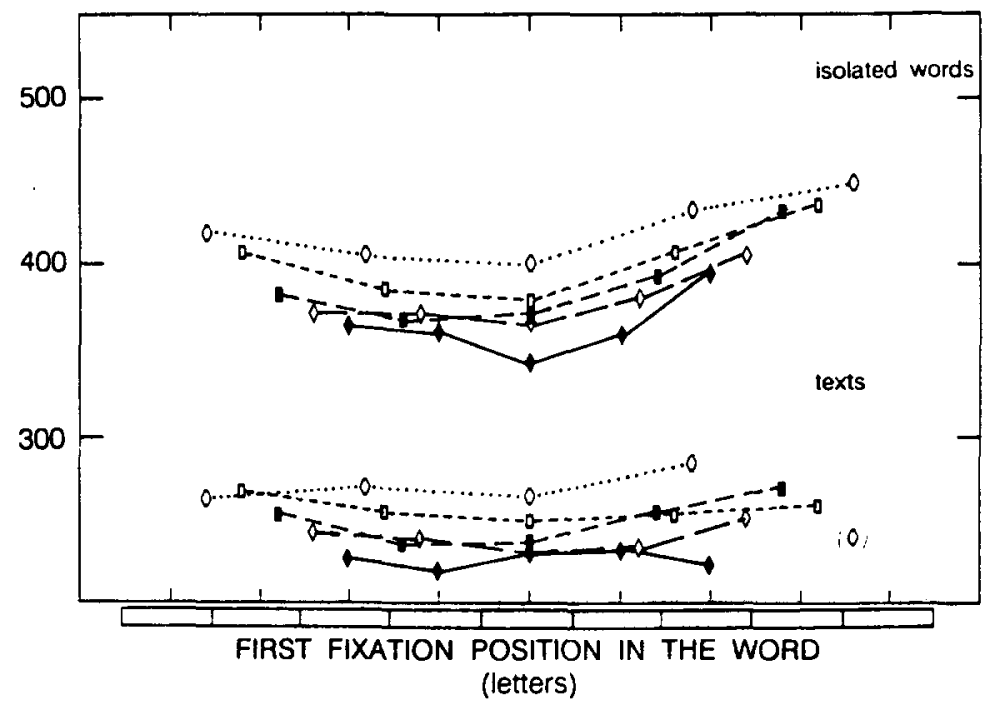

(b)

Figure 2. Probability of refixating the word (a) and gaze duration on the word (b; in milliseconds) as a function of initial fixation position in the word (five zones, corresponding to word length divided by 5), for 5-, 6-, 7-, 8-, and 9-letter words, in the isolated-word-reading condition (upper curves) and the text-reading condition (lower curves). Data for high-, medium-, and low-frequency words have been collapsed together. The blocks below the abscissa symbolize the positions letters occupy in the case of 9-letter words. For all word lengths, the middle of the abscissa corresponds to the middle of the word. For 9-letter words, the data point for the fifth zone (in parentheses) corresponds to a case in which only $4 \%$ of the fixations occurred.

9-letter words, a significant effect appeared, with refixation probability being higher for the lower frequency words $[F(2,32)=17.031, p<.0005]$.

Gaze duration. The effects on gaze duration closely follow those observed for refixation probability, with one interesting exception-namely, the effect of word frequency.

The effect of initial fixation position on gaze duration can be seen in Figure 2b (top curves). As for refixation probability, for all word lengths, gaze duration on the 
PROBABILITY OF REFIXATING A WORD

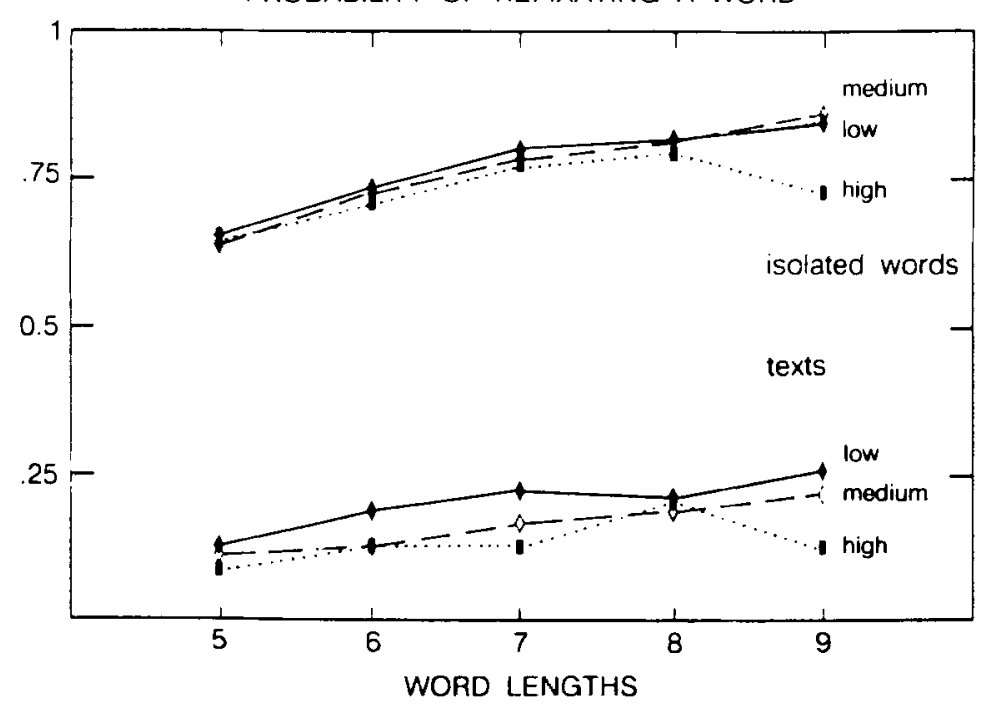

(a)

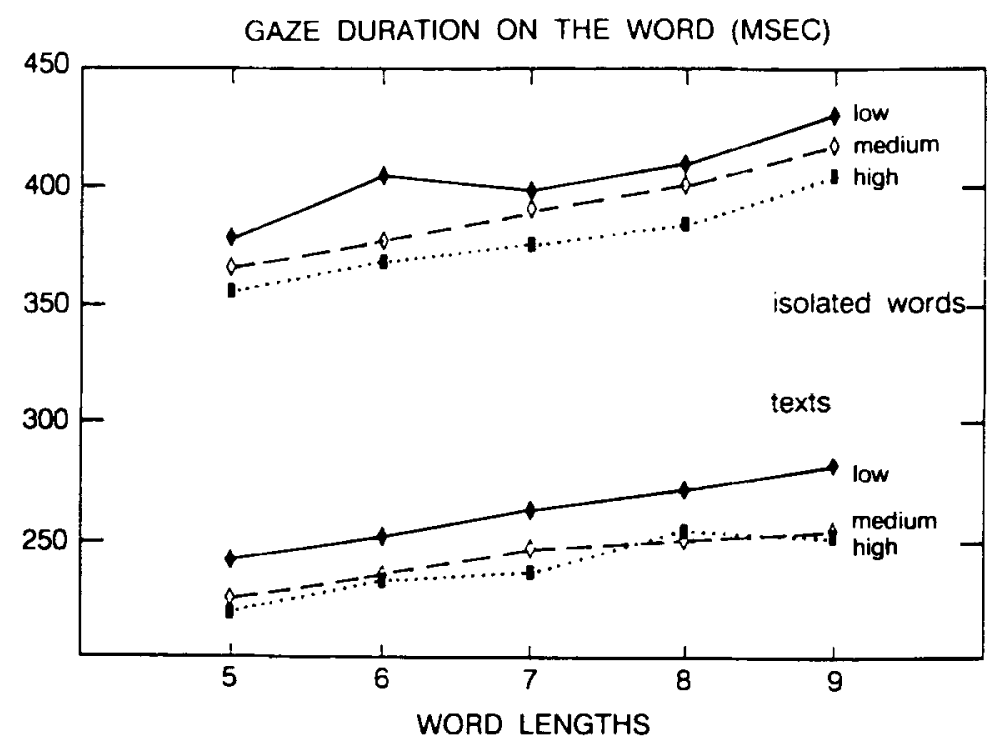

(b)

\begin{abstract}
Figure 3. Probability of refixating the word (a) and gaze duration on the word (h; in milliseconds) as a function of word length $(5,6,7,8$, and 9 letters), for low-, medium-, and high-frequency words, in the isolated-word-reading condition (upper curves) and the text-reading condition (lower curves). Data have been collapsed across all initial fixation zones.
\end{abstract}

word was shortest near the middle of the word, with first fixation position having a significant effect $[F \mathrm{~s}(4,64) \geq$ $6.329, p<.0005$, for each of the word lengths of 5,6 , 7,8 , and 9 letters].

The effect of word length on gaze duration was also significant $[F(4,64)=33.362, p<.0005]$, as it was for refixation probability. This is seen from the vertical displacement of the different curves in Figure $2 b$ (top). In
Figure $3 b$ (top curves), it can be seen that, overall, gaze duration increases by a little more than $10 \mathrm{msec}$ per letter of word length.

Contrary to the situation for refixation probabilities, there was a global effect of word frequency on gaze duration. Figure $3 b$ (top curves) shows that for all word lengths, gaze duration increases systematically as words become rarer $[F(2,32)=7.976, p<.0005, F(2,32)=11.795$, 
$p<.0005, F(2,32)=4.293, p<.025, F(2,32)=8.205$, $p<.005$, and $F(2,32)=8.106, p<.005$, for 5-, 6-, 7-, 8-, and 9-letter words, respectively].

Earlier work (O'Regan \& Lévy-Schoen, 1987) on the effect of word frequency and length had suggested that the optimal landing position for long, rare words was displaced more toward the beginning of the words than it was for frequent words. However, in the present experiment, word length had no effect on the location of the optimal landing position relative to the word's middle. As concerns the word-frequency effect on the optimal landing position, when gaze duration curves such as those in Figure $2 b$ (top) were plotted separately for each frequency and length, even though the U-shaped nature of the curves was obvious, no clear differences in the positions of the minima of the curves relative to the words' middles as a function of word frequency were apparent. Note, however, that we cannot totally exclude the possibility that there might be differences as a function of word frequency, because for a given word length, the number of high- and low-frequency words contributing data to a particular initial fixation position was small and could not be perfectly counterbalanced in the experimental design (cf. Table 3).

\section{Text Reading}

Probability of refixating a word. For all word lengths, the probability of refixating a word depended on the initial fixation position in the word (Figure 2a, bottom curves): for 5-, 6-, 7-, and 8-letter words, when the eye first fixated the middle of the word or slightly to the left of the middle, the probability of refixating was smaller than when the eye first fixated another position in the word. However, comparison with the upper curves in Figure 2a shows that the slopes of the curves were not as strong as they were for the isolated-word-reading condition. In addition, the effect was only significant for 6and 7-letter words $[F(4,64)=056$, n.s., $F(4,64)=5.726$, $p<.001, F(4,64)=3.352, p<.025$, and $F(4,64)=$ 1.134 , n.s., for 5-, 6-, 7-, and 8-letter words, respectively]. For 9-letter words, refixation probability dropped suddenly to a low value for the rightmost zone in the word $[F(4,64)=2.458, p<.10]$, but this is probably a quirk due to the fact that only very few fixations $(4 \%)$ occurred in this zone.

In addition to the weaker slopes of the curves, another difference with the situation for isolated words is the fact that for all word lengths, the optimal landing position where refixation probability was smallest was slightly shifted to the left.

The effect of word length on refixation probability was globally significant $[F(4,64)=12.570, p<.0005]$, although it appeared to be more variable (Figure 2a, bottom) and was strongly present only for medium-frequency words (Figure 3a, bottom).

Contrary to the case for isolated words, refixation probability depended on word frequency (Figure 3a, bottom curves); the effect was significant for three of the five word lengths $[F(2,32)=1.430$, n.s., $F(2,32)=3.831$, $p<.05, F(2,32)=5.755, p<.01, F(2,32)=.171$, n.s., and $F(2,32)=6.026, p<.01$, for 5-, 6-, 7-, 8-, and 9-letter words, respectively].

Gaze duration. Gaze duration on the word depended only slightly on the eye's first fixation position; this is shown by the bottom curves in Figure $2 b$, which reveal the same tendency as do those for refixation probability (Figure 2a, bottom curves), with a suggestion of an optimal landing position, but in a weaker fashion. The effect of the initial fixation position on gaze duration was not significant for any of the five word lengths $[F(4,64)$ $=.649$, n.s., $F(4,64)=.713$, n.s., $F(4,64)=2.359$, $p<.10, F(4,64)=1.010$, n.s., and $F(4,64)=2.122$, $p<.10$, for 5-, 6-, 7-, 8-, and 9-letter words, respectively]. The shifting to the left of the gaze-duration minimum as compared with what occurred in the isolatedword-reading condition was not so clear as it was for the refixation probability curves; the curves' minima extended over a larger zone.

As for isolated words, a significant global effect of word length on gaze duration was observed $[F(4,64)=13.397$, $p<.0005$ ], which can be seen from the fact that the curves for different lengths are vertically displaced (Figure $2 b$, bottom). Figure $3 b$ (bottom curves) shows the effect averaged over all initial fixation positions, and it is seen to be of the same order as for isolated wordsthat is, a little more than $10 \mathrm{msec}$ per letter of word length.

The effect of word frequency observed in the isolatedword-reading condition continued to exist in the present text-reading condition, but in a different manner (Figure $3 \mathrm{~b}$, bottom curves); gaze duration for high-frequency words seemed to be similar to gaze duration for mediumfrequency words, whereas for low-frequency words, gaze duration was systematically longer. This effect was significant $[F(2,32)=3.77, p<.05, F(2,32)=4.978$, $p<.025, F(2,32)=12.538, p<.0005, F(2,32)=$ $6.606, p<.0005$, and $F(2,32)=7.281, p<.005$, for 5-, 6-, 7-, 8-, and 9-letter words, respectively]. It resembled the effect of word frequency on refixation probability for text reading.

As for isolated words, word length and frequency seemed to have no clear effect on the exact location of the optimal landing position relative to the middle of words. Again, however, because of imperfect counterbalancing of the length $\times$ frequency $\times$ first fixation zone cells in the design, this conclusion must be treated with caution.

\section{DISCUSSION}

The present results have again confirmed the existence of an optimal landing position phenomenon in isolated words (cf. O'Regan \& Lévy-Schoen, 1987; O'Regan et al., 1984). The increase in refixation probability when subjects are not first fixating the optimal landing position is similar in our experiment and in the cited experiments. Moreover, the penalty in gaze duration for not starting 
to fixate the optimal position is of the same order in all the experiments; it equals approximately $20 \mathrm{msec}$ per letter of deviation from the optimal position.

As to the question of whether the optimal landing position phenomenon continues to exist in text reading, our refixation probability data, taken on their own, provide some positive evidence; the optimal landing position effect is statistically significant for some word lengths. Moreover, as discussed below, McConkie et al. (1989) found similar results, and it is on the basis of this converging evidence that we feel justified in concluding that the optimal landing position persists in text reading, at least for refixation probability.

In Figure 4a-4d, data from McConkie et al.'s (1989) experiment have been plotted together with our data for 5-, 6-, 7-, and 8-letter test words in the text-reading condition. Both sets of curves show an optimal position, with similar penalties for not first fixating the optimal position. However, for words of 5, 6, and 7 letters, in our case, the probability of refixating the word is higher on the right of the optimal position than on the left, whereas the situation is reversed in the case of McConkie et al.'s data. In order to understand the shape of their curves, McConkie et al. asked whether they could be explained simply by assuming that saccade size was approximately constant around 5 or 6 character spaces. If that were the case, then, when the eye first fixated near the beginning of a word, the probability that the following fixation would still be in the word would be higher than when the eye first fixated the middle or end of the word. Indeed, this was suggested by the shape of their curves. However, a statistical analysis allowed McConkie et al. to reject this hypothesis. With respect to our results, the hypothesis is much less pertinent, since we observed that the probability of refixating the word when the eye initially fixated the end of the word was high, whereas the constant saccade size hypothesis would have predicted the contrary. The fact that at word ends our curves tend to rise higher than McConkie et al. 's curves could be related to a difference in the morphological role of word ends in French and in English.

As concerns our gaze-duration data for the text-reading condition, the optimal landing position phenomenon is less evident than it is for the refixation probability data, being present only as a trend. Our hypothesis is that, in text reading, gaze duration depends not only on refixation probability, but also on individual fixation durations. This might cause the optimal landing position effect for gaze duration to become more noisy, by virtue of the increased variability of individual fixation durations related to factors present during text reading. We will return to this hypothesis later, when we examine individual fixation durations in the two reading conditions.

The optimal landing position phenomenon having been confirmed for the reading of both isolated words and texts (at least with respect to refixation probability), we now consider how the phenomenon differs in the two reading situations. Indeed the most important finding in the present experiment was certainly the large difference between the optimal landing position phenomenon in the isolatedword-reading and in the text-reading conditions. First, the increase in refixation probability when the eye did not first fixate the optimal landing position was weaker in the text-reading than in the isolated-word-reading condition. Second, the exact location of the optimal landing position in words was shifted to the left of the words' middles in the text-reading condition, rather than being close to the center as it was in the isolated-word-reading condition. In the following paragraphs, we shall do some further analyses in order to understand the weakening of the optimal landing position phenomenon and its leftward shift during text reading.

\section{The Question of Calibration Accuracy}

Before we consider some more interesting possibilities to explain the weakening of the optimal landing position effect in the text-reading situation, it is worth asking whether the weakening might simply be due to the fact that calibration accuracy was not so good in the textreading situation. It is true that this could have had the effect of mixing data from different fixation positions and thus of making the location of the minimum in each data curve less clear. However, calibration accuracy should not affect the slope of the data curves in the regions far from the minima of the curves, particularly for long words. Yet these slopes were consistently weaker than they were in the isolated-word-reading condition. In addition, McConkie et al.'s (1989) data on refixation probability in reading were gathered with more accurate equipment than ours and still showed slopes similar to ours (Figure 4). We shall therefore assume that the weakening of the optimal landing position effect in the textreading condition is not an artifact.

\section{The Effect of Parafoveal Preprocessing on the Strength of the Optimal Landing Position Phenomenon}

There are many differences between isolated-word reading and text reading that could explain the weakening of the optimal landing position phenomenon in text reading. First, in the isolated-word-reading situation, the eye was stationary, and it directly fixated the word rather than engaging in a sequence of forward saccades and fixations as in text reading. Second, parafoveal preprocessing and, third, linguistic context were available in the text-reading condition. These three factors could have decreased the probability of refixating the words and decreased fixation durations independently of the eye's first fixation position in the word.

Although it was not manipulated in this experiment, the effect of parafoveal preprocessing can be studied a posteriori. To do this, the text-reading data were reanalyzed as a function of the duration and position relative to the test word of the fixation that preceded the saccade into the test word. Data were divided into three classes, depending on the launch site of the saccade leading into the test 
PROBABILITY OF REFIXATING A WORD 5 - letter words

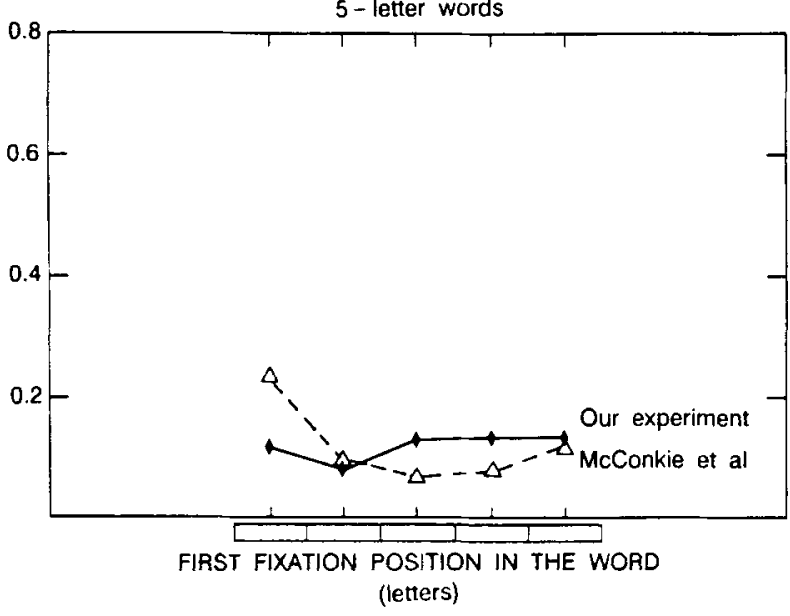

PROBABILITY OF REFIXATING A WORD

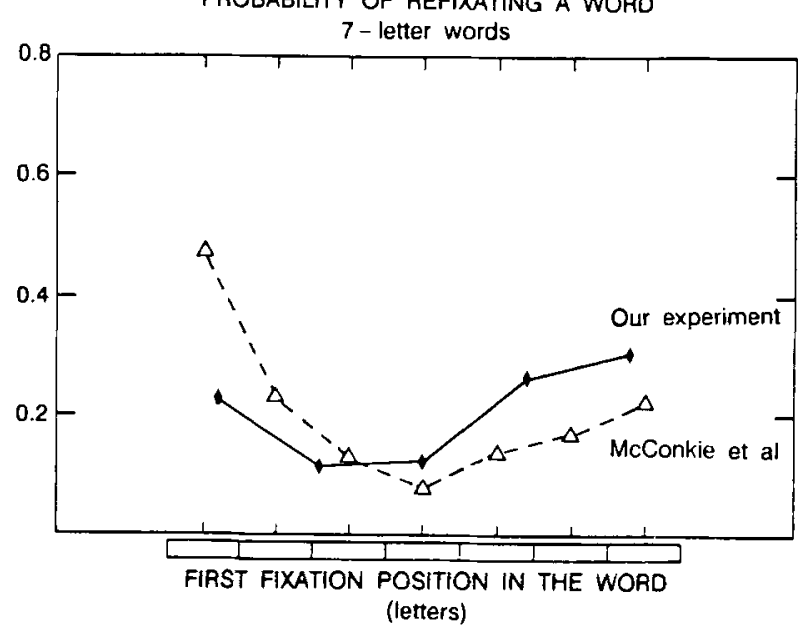

PROBABILITY OF REFIXATING A WORD 6 - letter words

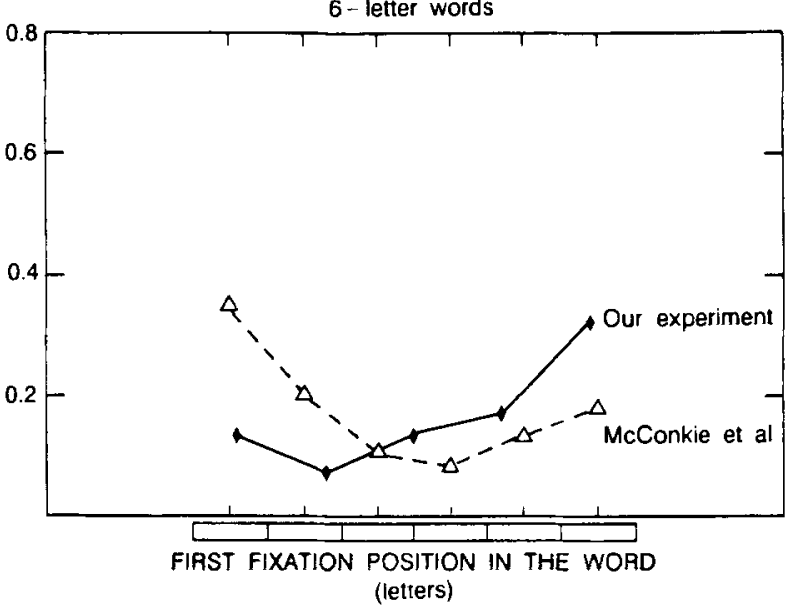

PROBABILITY OF REFIXATING A WORD 8 -letter words

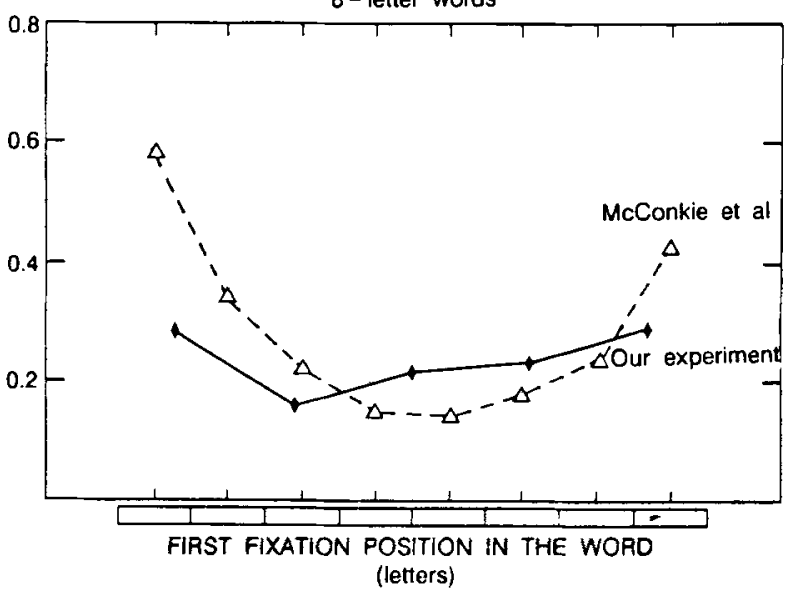

Figure 4. Probability of refixating the word as a function of initial fixation position in the word, in the text-reading condition in our experiment (solid lines) and in McConkie et al.'s (1989) experiment (dashed lines), for words of 5, 6, 7, and 8 letters. Although McConkie et al. also measured refixation probability for fixations occurring in the space before the word, only data for fixations occurring within the word are presented here.

word. We defined three launch sites: $1-5$ letters from the test word, 6-10 letters from the test word, and 11 or more letters from the test word. We reasoned that these zones would correspond to regions where progressively less parafoveal information about the test word will have been gathered (although of course this measure was confounded with saccade size). However, the data for the most distant zone was much scarcer than for the other two zones, so only these were ultimately retained (near and far launch sites). No analyses of variance could be done on these data, because of the widely different numbers of data points for the two zones.

For all word lengths except 9 letters, a systematic effect of launch site was observed on refixation probability. As can be seen in Figure 5, the probability of refixating when the eye was launched from near the test word (that is, when much parafoveal preprocessing was possible) was smaller than when it was launched far from the test word, and less parafoveal preprocessing was possible. This effect, which we assume is attributable to parafoveal preprocessing, seemed to be greater when the eye landed at the ends of words. However, the difference was small, and even for near launch sites (and strong preprocessing) there was still a strong optimal landing position effect. Thus, although parafoveal preprocessing has a global effect, it seems not to change the slopes of the refixation probability curves; parafoveal preprocessing seems not to be a very important factor in determining the weakening of the optimal landing position effect for refixation probability. Note that a similar conclusion was reached 
PAOBABILITY OF REFIXATING A WORD 5 - letter words

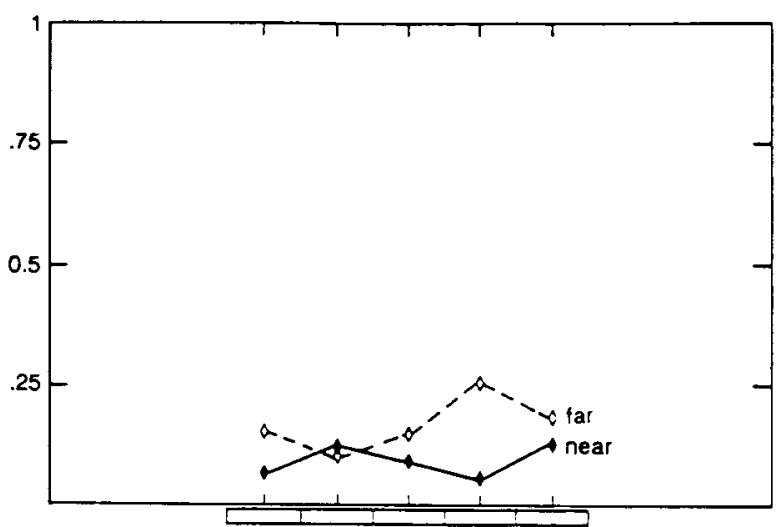

FIRST FIXATION POSITION IN THE WORD (letters)

PROBABILITY OF REFIXATING A WORD 7 - letter words

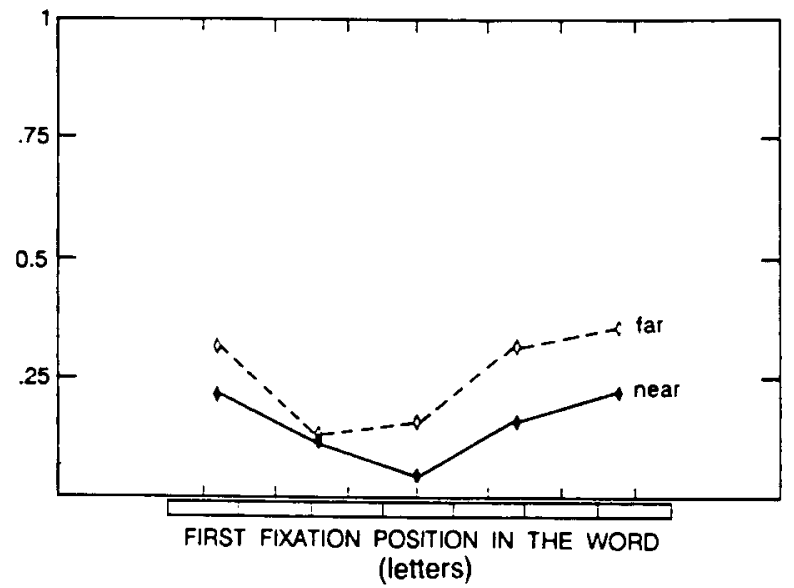

PROBABILITY OF REFIXATING A WORD 6 - letter words

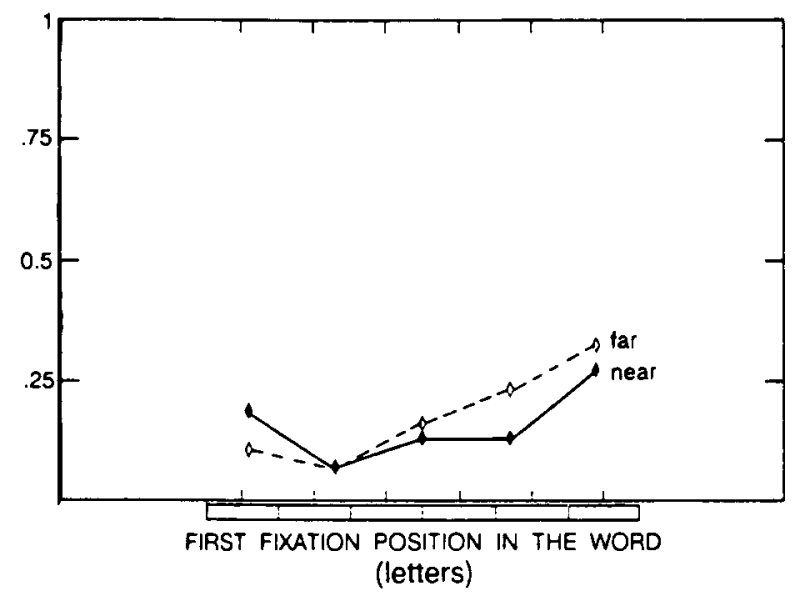

PROBABILITY OF REFIXATING A WORD 8 - letter words

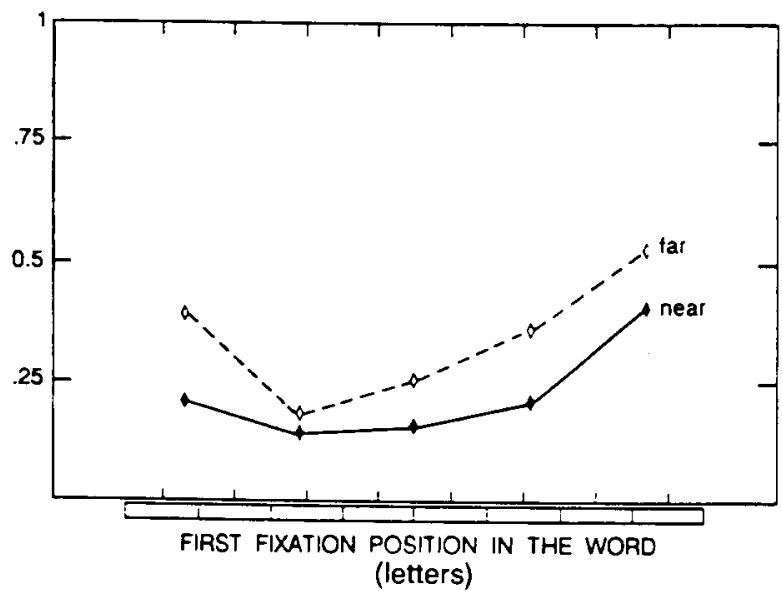

PROBABILITY OF REFIXATING A WORD

9 - letter words

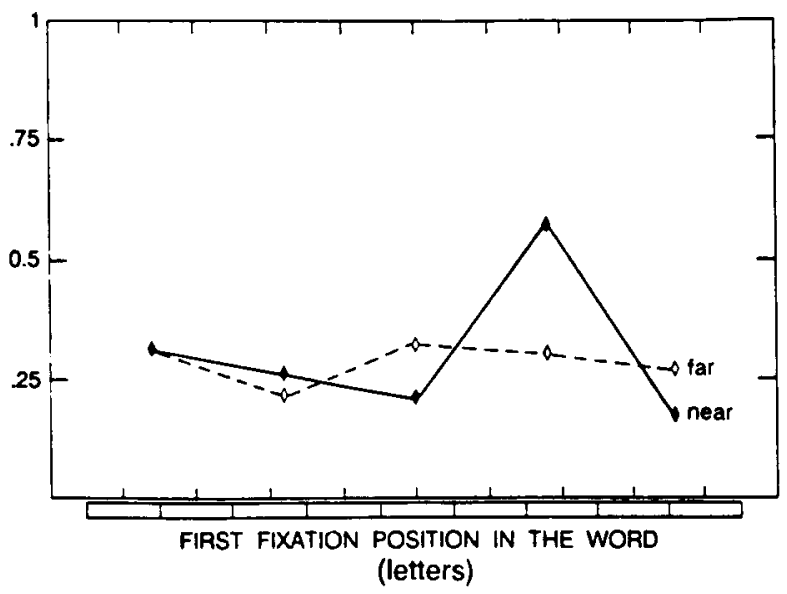

Figure 5. Probability of refixating the word in the text-reading condition as a function of the launch site of the saccade leading into the test word (near $=1-5$ letters left of the test word, and far = 6-10 letters left of the test word). The different graphs correspond to data for words of $5,6,7,8$, and 9 letters. 
in an isolated-word-reading study by Vitu and O'Regan (1988, in press), in which availability of parafoveal information surrounding isolated words was directly manipulated and there was no confounding with saccade size.

A possibility for explaining why parafoveal preprocessing does not influence the optimal landing position effect might be that parafoveal preprocessing takes time. Thus, its effect might only be strong when the fixation preceding the test word has a long duration. We checked for this possibility by subdividing the data according to whether the fixation prior to landing in the test word was longer or shorter than the median fixation duration. Contrary to our expectations, the effect of parafoveal preprocessing on refixation probability was actually stronger when the fixation duration preceding the test word was short (less than $215 \mathrm{msec}$ ) than when it was long. However, the strength of the optimal landing position effect was not affected by the duration of the fixation preceding the test word.

As concerns the gaze-duration data (which are not presented here), although parafoveal preprocessing had the global effect of shortening gaze durations overall, it did not seem to affect the strength of the optimal landing position for 6-, 7-, and 8-letter words; the optimal landing position phenomenon was not different for the two launch site conditions. This result is quite similar to that of Vitu and O'Regan (1988, in press). In the case of isolated words, we also found a global parafoveal preprocessing effect on gaze duration; however, there was no weakening of the optimal landing position phenomenon with parafoveal preprocessing. Thus, the strong weakening of the optimal landing position effect observed for gaze durations in our text-reading condition cannot be attributed to the effect of parafoveal preprocessing.

From the preceding analysis, and in agreement with the conclusions reached in earlier studies (Vitu \& O'Regan, 1988 , in press), only a part of the weakening of the optimal landing position phenomenon during text reading could be explained by parafoveal preprocessing. It must therefore be that most of the weakening of the optimal landing position phenomenon during text reading comes from the effect of factors such as reading rhythm and linguistic context, and from their interactions with parafoveal preprocessing. These factors could decrease the probability of refixating the word whatever the initial fixation position, and thus they could affect gaze durations (Balota et al., 1985) and/or individual fixation durations (Ehrlich \& Rayner, 1981; Zola, 1984).

\section{The Leftward Shift of the Optimal Landing \\ Position for Words in Texts}

One would have predicted that parafoveal preprocessing would mainly reduce the necessity of processing the first few letters of words. The most efficient place to fixate within a word during text reading would therefore be expected to be shifted to the right, and the optimal landing position should be further to the right than for isolated words, where no parafoveal preprocessing was pos- sible. Actually, however, the optimal landing position was observed to be shifted to the left rather than to the right during text reading.

Although calibration accuracy was less good in the textreading condition of the present experiment, there is no reason for this inaccuracy to have systematically caused an apparent systematic leftward shift of the optimal landing position. In fact, had there been any such artifact, it would have manifested itself in a shift of the observed distributions of positions where the eye landed in words. And yet, as noted above (Figure 1), these distributions of preferred landing positions were very similar to those found, using more accurate equipment, by McConkie et al. (1988). Thus, the leftward shift of the optimal landing position during text reading compared with reading isolated words in our study still needs explaining.

A closer look at Figure 5 does suggest that parafoveal preprocessing had the expected effect. If we neglect 5letter words, where the curves are noisy, and if we neglect the fifth zone of 9-letter words, which seems to be a quirk due to there being very little data for this landing zone, then in all cases the effect of parafoveal preprocessing (as measured by the difference between the curves for far and near launch sites) is to shift the optimal landing position to the right (near the word's middle) or at least to flatten the curves near the center. This observed rightward shift of the optimal position with parafoveal preprocessing is similar to the one observed by Vitu and O'Regan (1988, in press) for long words.

Nevertheless, despite the fact that parafoveal preprocessing is shifting the optimal landing position to the right, overall in text reading the optimal landing position seems to be shifted to the left as compared with what occurs for the reading of isolated words. We have no explanation for this at the moment, other than to suppose that the effect may be related to specific oculomotor strategies adopted by our readers in the text-reading condition.

\section{The Preferred and the Optimal Landing Positions}

Another interesting fact may be related to the unexpected leftward shift of the optimal landing position. The optimal landing position in words is defined in terms of the smallest refixation probability (or shortest gaze duration). However, there is no guarantee that the eye actually prefers to land at the optimal position.

Figures 1a-1e show the histograms of positions where the eye actually landed in the test words of the present text-reading condition. In agreement with earlier studies (Dunn-Rankin, 1978; McConkie et al., 1988; Rayner, 1979), the preferred landing position seems to fall within a fairly wide zone between the beginning and the middle of a word, the most frequently fixated position being the word's middle or a little to the left of it. This seems also to be approximately where the optimal landing position is located.

Interestingly, McConkie et al. (1989) found an optimal landing position in reading which was at word center, 
rather than shifted to the left (Figure 4). However, the preferred landing position in their studies was the same as it was in ours-that is, at, or just to the left of, the center. It seems as if readers' strategies in our study were better adapted to the optimal landing positions than they were in McConkie et al.'s studies.

Perhaps this difference between the two experiments (i.e., the difference concerning the location of the preferred landing position in words relative to words' optimal landing positions) results from differences in the abilities of the readers, or from different scanning strategies used by the readers (for example, in McConkie et al.'s [1989] experiment, some lines of texts contained erroneous words or nonwords, which was not the case in our experiment; this difference in text construction could have invoked different scanning strategies). It could also result from the construction of the text material (McConkie et al.'s words being globally more frequent than ours). This hypothesis cannot be asserted or rejected here, since in the present experiment no clear conclusion arose from the examination of the effect of word frequency on the location of the optimal landing position. A final interesting possibility for explaining the difference between the two experiments in the location of the preferred landing position relative to the optimal landing position might be related to the difference in language. It may be that, on the average, English words' optimal landing positions are nearer words' ends than French words' are.

The correspondence in our experiment between the most frequent landing position in words and the optimal landing position suggests that the strategy readers use is to aim for the optimal landing position. However, Figures la-le show that there remained a large proportion of cases in which the eye landed at nonoptimal positions. One possibility for explaining this may be related to the influence of factors such as parafoveal preprocessing or linguistic context. When these are not available, the eye may be unable to localize where the optimal landing position in the next word is. Another possibility is that oculomotor factors may prevent the eye from attaining the position it is aiming for (Coëffe \& O'Regan, 1987; Findlay, 1981); alternatively, it may be more efficient in reading to trigger saccades quickly and inaccurately, and to risk the consequences of inaccurate positioning in words, rather than to delay triggering in order to accurately attain an aimed-for position (see O'Regan, 1990, for further discussion of this). All of these arguments could explain why, in McConkie et al.'s (1989) experiment, readers' strategies were not adapted to the optimal landing position. The optimal landing position in their case being nearer a word's end, the eye could not saccade accurately to this position because of oculomotor constraints.

A consequence of the variability of actual landing position around the optimal landing position is that a precise knowledge of refixation probability as a function of initial landing position for all kinds of words (length, frequency, predictability from context) is desirable if one is to understand oculomotor behavior.

\section{The Importance of Within-Word Tactics in Understanding the Variability \\ of Fixation Durations}

In order to understand eye-movement behavior during reading, many researchers have studied the influence of visual and linguistic factors on gaze durations and individual fixation durations. However, as was shown for the case of isolated words, gaze durations and individual fixation durations seem to depend strongly on tactics within words (whether one or more fixations are made on the word; cf. O'Regan \& Lévy-Schoen, 1987). If within-word tactics are as important during text reading as during isolated-word reading, this would mean that all analyses of gaze durations and individual fixation durations during text reading done without taking into account within-word tactics would have to be questioned. We will therefore present some further analyses of our data, which were done to test the influence of within-word tactics on these measures in text reading.

O'Regan and Lévy-Schoen (1987) found in the case of isolated words that the decrease of gaze duration when the eye was on the optimal landing position came essentially from a decrease of the probability of refixating the word, gaze duration being systematically shorter when the eye made only one fixation than when the eye made two or more fixations on the word. Gaze duration in each case (one, or two, or three fixations on the word) was, however, only slightly affected by the eye's initial fixation position. Moreover, individual fixation durations depended directly on the pattern of fixation on the word; first fixation duration was longer when it was the only fixation than when it was followed by a second one.

In the present experiment, gaze duration and individual fixation durations were measured as a function of the fixation pattern on the word. For the isolated-word condition, for all word lengths, gaze duration was shorter when there was only one fixation than when two fixations $o c-$ curred, the difference being about $50 \mathrm{msec}$ (Figure 6a). When there was only one fixation, gaze duration seemed to depend slightly on initial fixation position; however, since the one-fixation tactics were not very frequent in the isolated-word condition, particularly when the eye did not first fixate the optimal landing position, we cannot be very sure of the form of the curves. When two fixations occurred, gaze duration was approximately constant except in the cases where the eye first fixated a word's end. Further analyses (summarized in Figure 7) of individual fixation durations indicated that first and second fixation durations in two-fixation tactics were overall shorter (by $150 \mathrm{msec}$ ) than fixation durations in onefixation tactics. Moreover, when two fixations occurred on the word, first and second fixation durations depended strongly on first fixation position, and first fixation duration was longer than second fixation duration. The precise pattern involved a tradeoff similar to that observed by O'Regan and Lévy-Schoen (1987): When the first fixation was far from the optimal landing position, its duration was shorter than when the first fixation was near the 
GAZE DURATION ON THE WORD (MSEC)

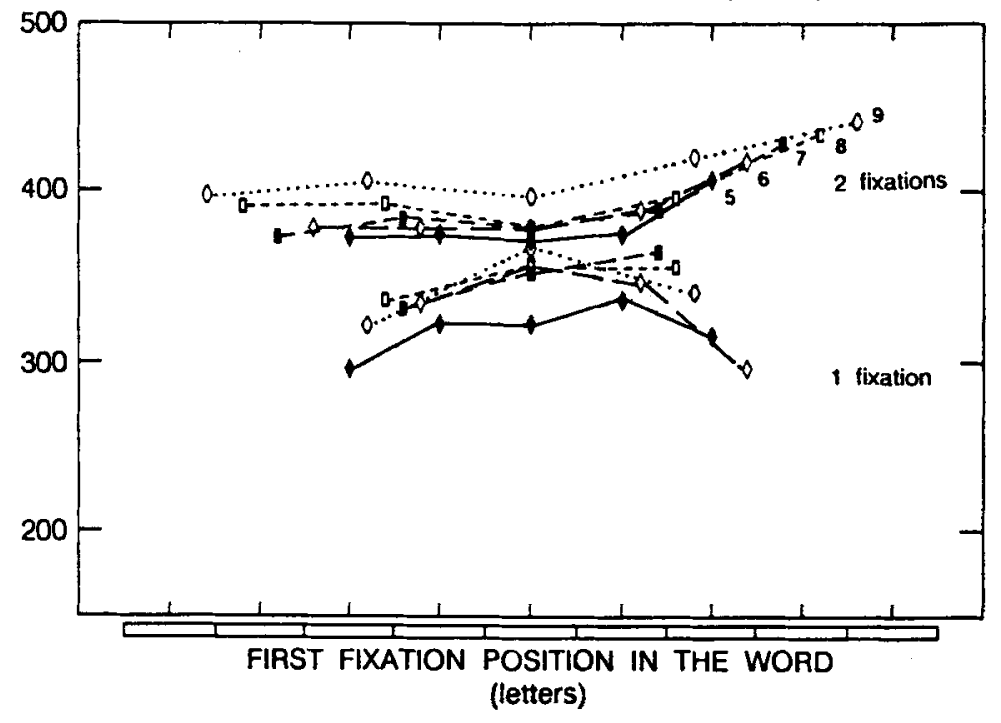

ISOUTED WORO READING CONOITION

(a)

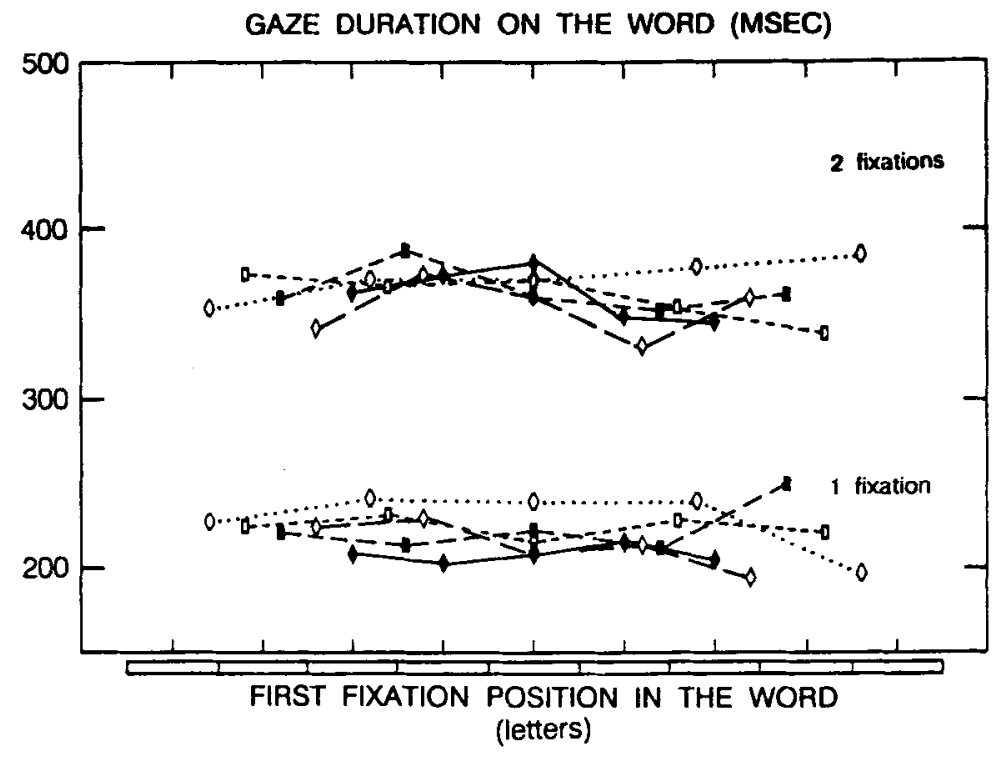

TEXT READING CONDITION

(b)

Figure 6. Gaze duration on the word (in milliseconds) as a function of first fixation position in the word for one-fixation (lower curves) and two-fixation (upper curves) tactics, for word lengths of $5,6,7,8$, and 9 letters, in the isolated-word-reading condition (a) and the text-reading condition (b). Data points where fewer than 10 out of 20 subjects contributed data were not plotted. 
optimal landing position, and this was reversed for the second fixation duration.

In the text-reading condition, for all word lengths, the difference for gaze durations between the single and double fixation tactics was much greater than it was with isolated words (Figure 6b); the penalty involved in making two fixations on the word rather than one fixation was approximately $140 \mathrm{msec}$. Gaze durations in each case seemed to be affected slightly by the initial fixation position in the word, particularly in the two-fixation tactics. However, contrary to what occurred in the isolated-word condition, fixation durations for one-fixation tactics were only slightly longer (by $40 \mathrm{msec}$ ) than individual first and second fixation durations for two-fixation tactics (these
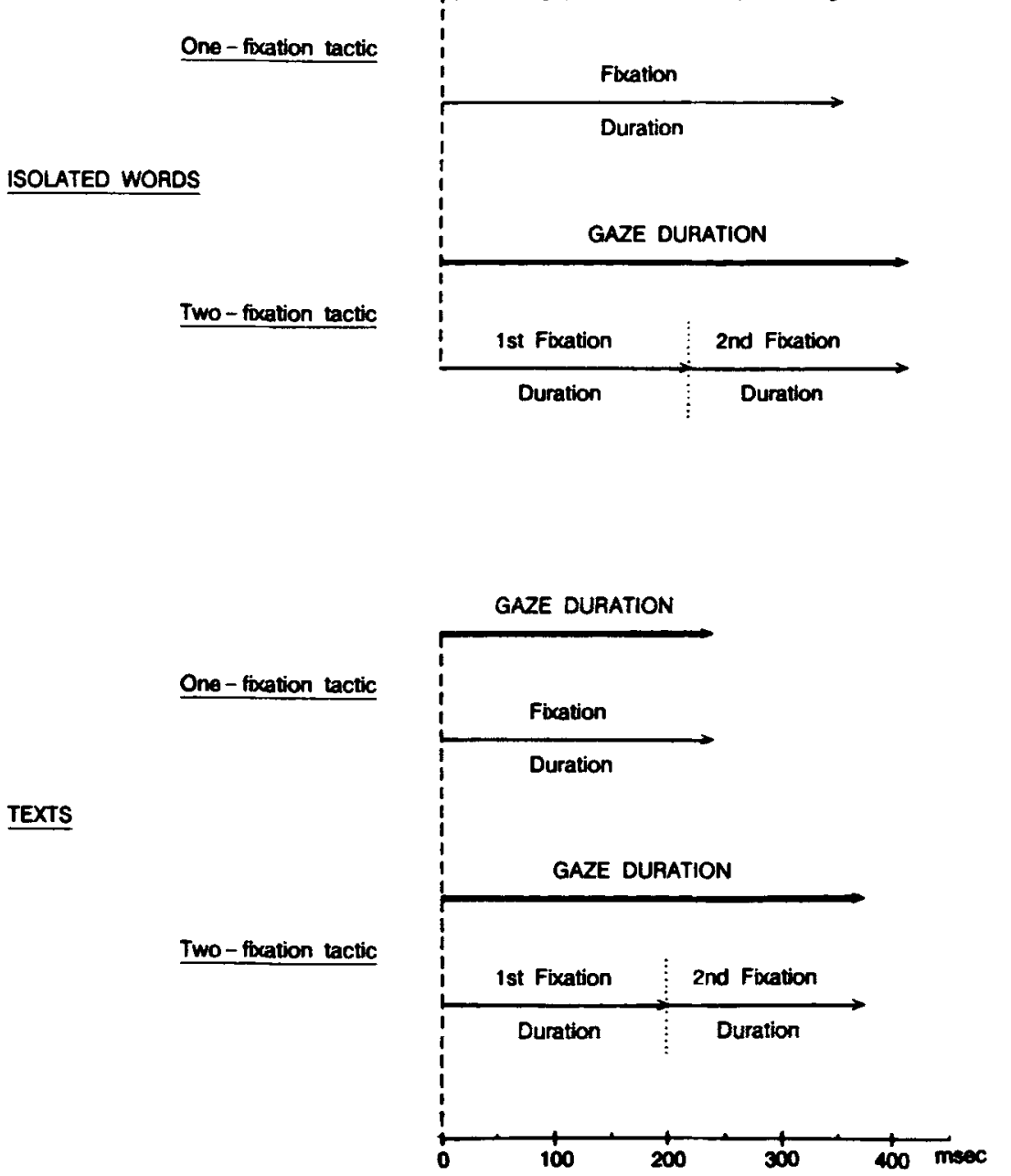

Figure 7. Summary of typical individual fixation-duration patterns for one-fixation and twofixation tactics, in the isolated-word-reading and the text-reading condition. For isolated words, gaze duration in the one-fixation tactic is about $50 \mathrm{msec}$ shorter than in the two-fixation tactic. For text reading, the difference between one- and two-fixation tactics is greater, about $140 \mathrm{msec}$. This is predominantly because the one-fixation tactic is of considerably shorter duration for text reading. In both isolated-word-reading and text-reading conditions, the two-fixation tactic has about the same gaze duration, with the first fixation being of slightly longer duration than the second. Note that in both conditions, first fixation duration in the two-fixation tactic is considerably shorter than in the one-fixation tactic (about 150 msec shorter for isolated words and about $\mathbf{4 0}$ msec shorter for text reading). Note also that in the two-fixation tactic, the relative durations of first and second fixations depend on the initial fixation position in the word. 
data are not shown here but are summarized in Figure 7). The individual fixation durations in the second case also followed the same tradeoff pattern of dependence on first fixation position as it did with isolated words. First fixation duration was also longer than second fixation duration.

Thus, the fact that the advantage of making only one fixation was stronger during text reading than during isolated-word reading came from the decrease of individual fixation durations in the one-fixation cases during text reading. This decrease in the fixation duration of single fixations, as well as the global decrease of gaze duration for the two-fixation tactics in the text reading condition (see Figures 6a-6b), is a good indication of the influence of text-reading factors on individual fixation durations. Since such factors are not controlled during text reading, they could have given rise to variability of fixation durations and thus rendered less clear the optimal landing position phenomenon for gaze-duration data in the text-reading condition. Since we have shown that parafoveal preprocessing did not change the effect of initial landing position on gaze duration, we assume that the reading factors involved are reading rhythm and linguistic context.

As was the case for O'Regan and Lévy-Schoen's (1987) data, all these results show how individual fixation durations are directly linked to patterns of fixation on the word and thus to initial fixation position and word length. Moreover, in two-fixation tactics, individual fixation durations depend directly on the eye's landing position in the word. Thus, refixation patterns and initial fixation position in words have to be considered if one is to understand the variability of eye fixation duration during reading.

\section{Comments on Word-Length and Word-Frequency Effects}

Two effects shown in the present experiment are of interest to psycholinguists. First, in both isolated-wordreading and text-reading conditions, both the probability of refixating the word and gaze duration increased strongly with word length. Second, a significant effect of frequency on gaze duration was noted in the isolated-wordreading condition; gaze duration for low-frequency words was longer than for high-frequency words. The same significant effect was also found for text reading. However, there was no effect of word frequency on refixation probability in the isolated-word-reading condition, and only a small effect in the text-reading condition.

It might be thought that the word-length effect could be due to a confounding of length with frequency, long words being globally rarer than short words. However, frequency means for low- and medium-frequency classes were approximately constant for all word lengths (see Table 1). Moreover, in the cases within the high-frequency class in which frequency means differ between two word lengths in such a way that the longer word is the more frequent (for example, high-frequency 5- and 6-letter words), we would expect that refixation probability or gaze duration would be smaller for the longer word if length was confounding frequency. However, the data show the contrary (Figure 3 ). Other evidence of the existence of a bona fide word-length effect comes from the fact that, for the two reading conditions, word length affected both the probability of refixating the word and gaze duration, whereas word frequency affected essentially gaze duration. So, word length exists as a causal factor independently of word frequency. This result is new, because until now, no experiments have been designed to show an effect of word length on refixation probability or gaze duration in a text-reading situation. O'Regan and Lévy-Schoen (1987) and Holmes and O'Regan (1987) showed the effect only in the case of isolated words. As concerns reading, only the reanalysis of Just and Carpenter's (1980) data by Kliegl, Olson, and Davidson (1982) suggested that word length could have an effect on gaze durations.

The effect of word frequency on gaze duration observed in our experiment confirms the word-frequency effect on gaze duration found by Rayner and Duffy (1986) and Inhoff and Rayner (1986). However, in those experiments, word length was only partially controlled, in the sense that each frequency class contained a number of different word lengths, even though the mean of these lengths was the same. Our word-frequency finding effect is therefore also new within the eye-movement literature.

The difference between the effect of word frequency on gaze duration and on refixation probability is compatible with O'Regan's strategy-tactics theory of eyemovement guidance in reading. According to this theory, refixation probability is primarily determined not by linguistic processing, but by factors available early in the fixation-that is, low-level visual factors. Gaze duration, however, can depend on linguistic factors such as word frequency, particularly in the case when two fixations occur or when a single fixation is long. As concerns the existence of the small word-frequency effect on refixation probability that was observed in the text-reading condition but not in the isolated-word-reading condition, this could have resulted from the fact that, in the text-reading condition, context facilitated visual encoding of the stimulus and allowed frequency effects to manifest themselves earlier in the fixation.

The effect of word frequency on gaze duration does not seem to interact with context; the size of this effect was comparable in the isolated-word-reading and the textreading conditions. This result is compatible with Schubert and Eimas' (1977) demonstration that context and frequency have additive effects in a lexical decision task. The observed stability of word-frequency effects across single word and text presentation is an important demonstration of the fundamental role played by word frequency in visual word recognition.

\section{CONCLUSION}

This experiment yielded two main groups of results that can help us understand the variability of fixation dura- 
tions observed in reading. The first group concerns the comparison of the optimal landing position phenomenon in isolated-word and in text reading, and it shows which factors influence within-word tactics (whether there will be one or more fixations on the word) during text reading. The second group shows how within-word tactics influence individual fixation durations during text reading.

The first group of results confirms the existence of the optimal landing position phenomenon for both isolatedword recognition (for refixation probability and gaze duration) and text reading (essentially, for refixation probability). Moreover, the optimal landing position effect in words included in texts differs from that observed for isolated words; during text reading, the effect is weaker and the exact location of the optimal landing position is shifted to the left within the word. Further analysis of our data, and data from other experiments, suggests that the weakening of the optimal landing position phenomenon is due to factors present during reading such as reading rhythm and linguistic context, rather than to the effect of parafoveal preprocessing; parafoveal preprocessing has only a global effect on refixation probability and gaze duration, and it does not affect the penalty incurred when the eye fails to fixate the optimal landing position. The observed leftward shift of the optimal landing position during text reading also does not seem to result from parafoveal preprocessing, since additional analyses demonstrated that this factor shifted the optimal position to the right.

The second group of results shows that gaze durations and individual fixation durations depend on within-word tactics; when a word is fixated twice, the durations of the individual fixations are shorter overall than they are when only a single fixation is made, and the sum of their durations is greater, so that total gaze duration shows a penalty as compared with the single fixation case. Moreover, analyses not reported in detail here showed that the precise pattern of durations of the fixations in the two-fixation tactic also depends strongly on the position initially fixated in a word.

The fact that the influence of within-word tactics on individual fixation durations is smaller in the text-reading condition than in the isolated-word-reading condition suggests the importance of factors such as reading rhythm and linguistic context on individual fixation durations. The large variability of fixation durations resulting from such influences may prevent the eye's landing position in words from affecting gaze durations as strongly as it affects refixation probability.

Therefore, our conclusion from this experiment is that individual fixation durations are determined strongly by within-word tactics, and thus by the eye's first landing position in words. In addition, individual fixation durations are influenced by the interaction of the initial landing position in words with reading rhythm and linguistic context.

Other interesting results concern the effect of word length on refixation probability and gaze duration, and the effect of word frequency on gaze duration, both of which persist during text reading. This seems to show that word length and word frequency influence word recognition at different levels than linguistic context does.

\section{REFERENCES}

Balota, D.A., Pollatsek, A., \&ayner, K. (1985). The interaction of contextual constraints and parafoveal visual information in reading. Cognitive Psychology, 17, 364-390.

BAlota, D. A., RAYNER, K. (1983). Parafoveal visual information and semantic contextual constraints. Joumal of Experimental Psychology: Human Perception \& Performance, 9, 726-738.

COËFFÉ, C., \& O'REGAN, K. (1987). Reducing the influence of nontarget stimuli on saccade accuracy: Predictability and latency effects. Vision Research, 27, 227-240.

Dunn-RAnkIN, P. (1978, January). The visual characteristics of words. Scientific American, 238(1), 122-130.

EhrLich, S. F., \& RAYNer, K. (1981). Contextual effects on word perception and eye movements during reading. Joumal of Verbal Learning \& Verbal Behavior, 20, 641-655.

FindlaY, J. M. (1981). Local and global influences on saccadic eye movements. In D. F. Fisher, R. A. Monty, \& J. W. Senders (Eds.), Eye movements: Cognition and visual perception (pp. 171-179). Hillsdale, NJ: Erlbaum.

Hogaboam, T. W. (1983). Reading patterns in eye movement data. In $\mathrm{K}$. Rayner (Ed.), Eye movements in reading: Perceptual and language processes (pp. 309-331). New York: Academic Press.

Holmes, V. M., \& O'RegaN, J. K. (1987). Decomposing French words. In J. K. O'Regan \& A. Lévy-Schoen (Eds.), Eye movements: From physiology to cognition (pp. 459-466). Amsterdam: North-Holland. INHOFF, A. W., \& RAYNER, K. (1980). Parafoveal word perception: A case against semantic preprocessing. Perception \& Psychophysics, 27, 457-464.

INHOFF, A. W. \& RAYNER, K. (1986). Parafoveal word processing during eye fixations in reading: Effects of word frequency. Perception \& Psychophysics, 40, 431-439.

Just, M. A., \& CARPEnTer, P. A. (1980). A theory of reading: From eye fixations to comprehension. Psychological Review, 87, 329-354.

Just, M. A., CARPENTER, P. A. (1987). The psychology of reading and language comprehension. Boston: Allyn \& Bacon.

KLIEGL, R., OLson, R. K., DAvidson, B. J. (1982). Regression analyses as a tool for studying reading processes: Comment on Just and Carpenter's eye fixation theory. Memory \& Cognition, 10, 287-296.

LimA, S. D., INHOFF, A. W. (1985). Lexical access during eye fixations in reading: Effects of word-initial letter sequence. Journal of Experimental Psychology: Human Perception \& Performance, 11, 272-285

MCClelland, J. L., \& O'Regan, J. K. (1981). Expectations increase the benefit derived from parafoveal visual information in reading words aloud. Joumal of Experimental Psychology: Human Perception \& Performance, 7, 634-644.

MCConkIE, G. W., KerR, P. W., Reddix, M. D., Zola, D. (1988). Eye movement control during reading: I. The location of initial eye fixations on words. Vision Research, 28, 1107-1118.

McConkIE, G. W., KerR, P. W., Reddix, M. D., ZolA, D., \& JACOBS, A. M. (1989). Eye movement control during reading: II. Frequency of refixating a word. Perception \& Psychophysics, 46, 245-253.

MoConkIe, G. W., UnderWOOD, N. R., Zola, D., \& Wolverton, G. (1985). Some temporal characteristics of processing during reading. Journal of Experimental Psychology: Human Perception \& Performance, 11, 168-186.

O'RegaN, [J.] K. (1979). Saccade size control in reading: Evidence for the linguistic control hypothesis. Perception \& Psychophysics, 25, 501-509.

O'RegAN, J. K. (1984). How the eye scans isolated words. In A. G. Gale \& F. Johnson (Eds.), Theoretical and applied aspects of eye movement research (pp. 159-168). Amsterdam: North-Holland. 
O'REgAN, J. K. (1989). Visual acuity, lexical structure, and eye movements in word recognition. In B. Elsendoorn \& H. Bouma (Eds.), Working models of human perception (pp. 261-292). London: Academic Press.

O'ReGAN, J. K. (1990). Eye movements and reading. In E. Kowler (Ed.), Reviews of oculomotor research: Vol. 4. Eye movements and their role in visual and cognitive processes (pp. 395-453). Amsterdam: Elsevier.

O'Regan, J. K., \& Lévy-SChoen, A. (1987). Eye movement strategy and tactics in word recognition and reading. In M. Coltheart (Ed.), Attention and performance XII: The psychology of reading (pp. 363383). Hillsdale, NJ: Erlbaum.

O'Regan, J. K., Lévy-Schoen, A., Pynte, J., \& Brugaillère, B. (1984). Convenient fixation location within isolated words of different length and structure. Journal of Experimental Psychology: Human Perception \& Performance, 10, 250-257.

RAYNER, K. (1975). The perceptual span and peripheral cues in reading. Cognitive Psychology, 7, 65-81.

RAYNER, K. (1979). Eye guidance in reading: Fixation locations within words. Perception, 8, 21-30.

RAYNER, K., DUFFY, S. A. (1986). Lexical complexity and fixation times in reading: Effects of word frequency, verb complexity, and lexical ambiguity. Memory \& Cognition, 14, 191-201.

Rayner, K., Inhoff, A. W., Morrison, R. E., Slowiczek, M. L., * Bertera, J. H. (1981). Masking of foveal and parafoveal vision during eye fixations in reading. Journal of Experimental Psychology: Human Perception \& Performance, 7, 167-179.
Rayner, K., McConkie, G. W., Ehrlich, S. (1978). Eye movements and integrating information across fixations. Journal of $E x-$ perimental Psychology: Human Perception \& Performance, 4, 529-544.

Rayner, K., Well, A. D., Pollatsek, A., \& Bertera, J. H. (1982). The availability of useful information to the right of fixation in reading. Perception \& Psychophysics, 31, 537-550.

SCHUBERT, R. E., \& Eimas, P. D. (1977). Effects of context on the classification of words and non-words. Journal of Experimental Psychology: Human Perception \& Performance, 3, 27-36.

VITU, F., \& O'REGAN, J. K. (1988). Effect of parafoveal preprocessing and reading rhythm on optimal landing position in words of different length and frequency. In G. Luer, U. Lass, \& J. Schallo-Hoffmann (Eds.), Eye movement research: Physiological and psychological aspects (pp. 286-292). Toronto: Hogrefe.

VITU, F., \& O'REGAN, J. K. (in press). Eye movement in reading: Optimal landing position and usefulness of peripheral preview. In D. Brogan (Ed.), The First International Conference on Visual Search Proceedings. Philadelphia: Taylor \& Francis.

ZOLA, D. (1984). Redundancy and word perception during reading. Perception \& Psychophysics, 36, 277-284.

(Manuscript received August 7, 1989; revision accepted for publication December 12, 1989.) 